\title{
Berberine reduces the occurrence of neonatal necrotizing enterocolitis by reducing the inflammatory response
}

\author{
YONG JING $^{1}$, FUDONG PENG ${ }^{2}$, YUFENG SHAN ${ }^{2}$ and JINGKAI JIANG ${ }^{1}$ \\ ${ }^{1}$ Department of Pediatric Surgery; ${ }^{2}$ Neonatal Intensive Care Unit, The Second People's Hospital of Liaocheng, \\ Linqing, Shandong 252600, P.R. China
}

Received February 2, 2018; Accepted August 9, 2018

DOI: $10.3892 /$ etm.2018.6871

\begin{abstract}
Necrotizing enterocolitis (NEC) is a life-threatening disease that occurs in premature infants. The aim of the present study was to investigate the effects of berberine, an isoquinoline alkaloid mainly used to treat digestive diseases, in a rat model of NEC. NEC models were established in newborn rats via inhalation of $\mathrm{N}_{2}$ for $90 \mathrm{sec}$ every $4 \mathrm{~h}$ and oral administration of $4 \mathrm{mg} / \mathrm{kg} /$ day lipopolysaccharides on days 0 and 1 . Berberine was administered via oral gavage. In the NEC model group, Toll-like receptor (TLR)4, nuclear factor NF- $\kappa \mathrm{B}(\mathrm{NF}-\kappa \mathrm{B})$, inducible nitric oxide synthase (iNOS), tumor necrosis factor (TNF)- $\alpha$, interleukin (IL)-6 and IL-10 were upregulated. Symptoms of NEC in the berberine intervention group were significantly relieved, with a clear reduction in the incidence of NEC compared with the NEC group. TLR4, NF- $\kappa B$, iNOS, TNF- $\alpha$, IL- 6 and IL-10 expression was decreased following berberine intervention. Furthermore, the expression of mucin-2 (MUC2) and RNA polymerase $\sigma$ factor SigA (SIgA) were decreased in the NEC model group and increased following berberine intervention, when compared with the untreated group. It was also demonstrated that the incidence of NEC was reduced following berberine administration, possibly owing to changes in the inflammatory responses. The results of the current study support a potential therapeutic role of berberine for the treatment of NEC.
\end{abstract}

\section{Introduction}

Necrotizing enterocolitis (NEC) is a serious, life-threatening disease in premature infants. The incidence of NEC has increased in spite of clinical developments (1-3). Additionally, the mortality rate of infants with NEC remains high ( 20-30\%),

Correspondence to: Dr Jingkai Jiang, Department of Pediatric Surgery, The Second People's Hospital of Liaocheng, 317 Yaokou Street, Linqing, Shandong 252600, P.R. China

E-mail: jiangjingkaii@163.com

Key words: necrotizing enterocolitis, berberine, necrotizing enterocolitis grade, inflammatory, Toll-like receptor 4 although the clinical outcome for many premature infants has improved (4). Severe NEC is often accompanied by the necrosis of the intestinal wall and perforation, as well as peritonitis, with a very high mortality rate (5). The severity of NEC varies from case to case any may affect the entire intestine $(6,7)$. Studies have demonstrated that various factors are associated with an increased risk of developing NEC; its pathogenesis has been explored using animal models in an attempt to develop more effective therapeutic strategies $(8,9)$. The latest studies have indicated that inflammatory cascades serve an important role in the pathogenesis of neonatal NEC $(10,11)$. Studies have hypothesized that NEC is caused by an uncontrolled inflammatory response induced by a characteristic intestinal bacterial colonization in premature infants $(12,13)$. Various inflammatory mediators, receptors and signal transduction pathways are involved in the pathophysiological processes of the disease; however, it remains unclear which factors are most crucial $(1,2)$. These factors are potential targets for regulating the prevention and treatment of NEC (14). According to some studies, an inflammatory cascade involving inflammatory cytokines serve a role in the onset and progression of NEC $(15,16)$. The aim of the present study was to develop a method for establishing an animal model of NEC to screen for effective treatment agents.

Berberine, an alkaloid widely used in traditional Chinese medicine to treat gastrointestinal infections, is an effective antimicrobial treatment with regulatory effects on glucose and lipid metabolism, as well as insulin resistance $(17,18)$. The anti-inflammatory and anti-oxidant effects of berberine and its suppression of gene transcription were recently reported (17). In addition, berberine maintains the junctions between intestinal mucosa (19). Furthermore, the efflux pump in the intestine mucosa promotes berberine distribution in the gastrointestinal epithelia (19). Therefore, the current study aimed to investigate how berberine affects NEC development in a rat model of NEC.

\section{Materials and methods}

NEC model establishment. A total of 60 newborn Sprague-Dawley (SD) rats (6-8 g; 30 Male, 30 Female) were obtained from pregnant SD rats (Shanghai SLAC Laboratory Animal Co., Ltd., Shanghai, China) on day 21 of gestation. Thereafter, newborn rats were fed with $0.1 \mathrm{ml}$ artificial milk 
(Pet-Ag, Inc., Hampshire, IL, USA) twice a day. Rats were housed at $30^{\circ} \mathrm{C}$ and $60 \%$ humidity with a 12-h light-dark cycle. NEC models were established via $\mathrm{N}_{2}$ inhalation for $90 \mathrm{sec}$ every $4 \mathrm{~h}$ and oral administration of $4 \mathrm{mg} / \mathrm{kg} / \mathrm{day}$ lipopolysaccharides (LPS; Sigma-Aldrich; Merck KGaA, Darmstadt, Germany) on days 0 and 1 (20). The present study was approved by the Animal Ethics Committee of The Second People's Hospital of Liaocheng (Linqing, China).

Animal grouping and berberine treatment. Newborn were weighed and randomly divided into three groups $(n=20)$. Rats in the normal control (control) group stayed with their mothers in the same cage following birth and were fed breast milk without any intervention. Neonatal rats in the NEC model (NEC) group were placed in an incubator $48 \mathrm{~h}$ after birth and established as the neonatal NEC rat models. Neonatal rats in the berberine intervention ( $\mathrm{NEC}+$ berberine) group were established as NEC rat models and berberine (Sigma-Aldrich; Merck KGaA) was administered twice a day for 4 days by gavage at a dose of $0.6 \mathrm{~g} / \mathrm{kg} /$ day dissolved in artificial milk.

NEC evaluation. Rats that developed distress (lethargy, abdominal distention and bloody diarrhea) or imminent death prior to $96 \mathrm{~h}$ were sacrificed to acquire the whole intestine. At $96 \mathrm{~h}$ later, remaining rats were sacrificed to obtain the intestines. Intestinal samples were fixed in $75 \%$ ethanol at $4^{\circ} \mathrm{C}$ for $16 \mathrm{~h}$ embedded in paraffin and sliced into 4-6- $\mu \mathrm{m}$-thick sections. These sections were stained using hematoxylin and eosin both for $1 \mathrm{~min}$ at room temperature. Histological evaluation of NEC was performed using light microscopy (magnification, x200) (Olympus BX51; Olympus Corporation, Tokyo, Japan). NEC severity was assessed according to the NEC scoring system (3). Histological changes in the intestinal architecture of rats with NEC were assigned an NEC grade: Grade N (normal), noseparation in the submucosa or lamina propria; grade L (low), slight submucosal and lamina propria separation; grade $\mathrm{M}$ (moderate), increased submucosal and lamina propria separation with edema of the submucosa; grade I (intermediate), severe separation of the submucosa and lamina propria; and grade $\mathrm{S}$ (severe), necrosis and loss of villi structure. Rats with grade M, I or S were deemed to have NEC.

ELISA. The rat intestinal tissues were homogenized in NP40 lysis buffer (Sigma-Aldrich; Merck KGaA) on ice and the homogenates were quantified using a BCA assay (Thermo Fisher Scientific, Inc., Waltham, MA, USA). Total intestinal proteins were assessed using an ELISA kit to evaluate the levels of IL-6 (cat. no. ab100712; Abcam, Cambridge, UK), IL-10 (cat. no. ab214566; Abcam), MUC2 (cat. no. LS-F4717; LifeSpan Biosciences, Inc., Seattle, WA, USA) and SIgA (cat.no. SE120114; Sigma-Aldrich; Merck KGaA) in the samples.

Reverse transcription-quantitative polymerase chain reaction $(R T-q P C R)$. Total RNA was extracted from intestinal tissues using TRIzol Reagent (Invitrogen; Thermo Fisher Scientific, Inc.) according to the manufacturer's protocol. Total RNA $(1 \mu \mathrm{g})$ was used to generate cDNA with SuperScript II reverse transcriptase (Invitrogen; Thermo Fisher Scientific, Inc.). qPCR was performed in triplicate using SYBR Premix Ex Taq (Takara, Dalian, Liaoning, China) and SsoFast ${ }^{\mathrm{TM}}$ Probes
Supermix (Bio-Rad Laboratories, Inc., Hercules, CA, USA) and a standard thermocycling procedure (35 cycles) was performed on a Bio-Rad CFX96 ${ }^{\mathrm{TM}}$ Real-time PCR System (Bio-Rad Laboratories, Inc.). The thermocycling conditions were as follows: $95^{\circ} \mathrm{C}$ for $2 \mathrm{~min}$; followed by 35 cycles of $95^{\circ} \mathrm{C}$ for $1 \mathrm{~min}, 60^{\circ} \mathrm{C}$ for $1 \mathrm{~min}$ and $72^{\circ} \mathrm{C}$ for $1 \mathrm{~min}$; then extension was performed at $72^{\circ} \mathrm{C}$ for $10 \mathrm{~min}$. The $2^{-\Delta \Delta \mathrm{Cq}}$ method was used to analyze the relative changes in gene expression (4). The primers used were as follows: TLR4 forward, 5'-GCATCATCT TCATTGTCCTTGA-3' and reverse, 5'-CTTGTTCTTCCT CTGCTGTTTG-3'; NF- $\kappa$ B forward, 5'-ATGGCAGACGAT GATCCCTAC-3' and reverse, 5'-CGGAATCGAAATCCC CTCTGTT-3'; iNOS forward, 5'-GAGGCCCAGGAGGAG AGAGATCCG-3' and reverse, 5'-TCCATGCAGACAACC TTGGTGTTG-3'; TNF- $\alpha$ forward, 5'-CCAGACCCTCAC ACTCAGATC-3' and reverse, 5'-CACTTGGTGGTTTGC TACGAC-3'; and $\beta$-actin forward, 5'-CTAAGGCCAACCGTG AAAAG-3' reverse, 5'-TACATGGCTGGGGTGTTGA-3'.

Western blot analysis. Total protein was extracted from ileal tissues with lysis buffer (Nanjing KeyGen Biotech Co., Ltd., Nanjing, China). The protein concentration was determined using a BCA assay. Proteins (50 $\mu \mathrm{g} /$ lane) were separated by $12 \%$ SDS-PAGE and transferred to a polyvinylidene difluoride membrane and blocked with $50 \mathrm{~g} / \mathrm{l}$ skimmed milk at room temperature for $1 \mathrm{~h}$. Primary antibodies against TLR4 (1:1,000; cat. no. ab13556), iNOS (1:1,000; cat. no. ab3523) and NF-кB (1:1,000; cat. no. ab32536; all Abcam) were incubated with the membranes overnight at $4^{\circ} \mathrm{C}$, following which membranes were incubated with an horseradish peroxidase-conjugated antibody (1:5,000; cat. no. 7071; Cell Signaling Technology, USA) at room temperature for $1 \mathrm{~h}$. The protein bands were visualized with a G-BOX imaging system (Syngene Europe, Cambridge, UK) using an ECL assay kit (Pierce; Thermo Fisher Scientific, Inc.). Western blotting results were analyzed by ImageJ software 1.8.0 (National Institutes of Health, Bethesda, MD, USA).

Statistical analysis. SPSS 17.0 software (SPSS, Inc., Chicago, IL, USA) was used for statistical analyses and all data are presented as the mean \pm standard deviation. One-way analysis of variance followed by a Tukey's post-hoc test was used to compare multiple groups and the least significant difference test was performed for pair-wise comparisons. $\mathrm{P}<0.05$ was considered to indicate a statistically significant difference.

\section{Results}

Berberine treatment decreases the incidence and severity of $N E C$. In the control, NEC and NEC + berberine groups, the incidence of NEC was 0,65 and $25 \%$, respectively (Fig. 1). A significant difference in the incidence rate of NEC between the NEC group and NEC + berberine group was observed (Fig. 2). Fig. $2 \mathrm{~B}$ presents the representative microscopic morphology of the intestinal samples. All rats in grade $\mathrm{M}$ or I exhibited bloody diarrhea (data not shown).

Berberine treatment decreases TLR4 expression in ileal tissues. In the NEC group, the expression of TLR4 mRNA and protein was significantly and markedly increased, respectively, at $48 \mathrm{~h}$ compared with the control group (Fig. 3). However, in 


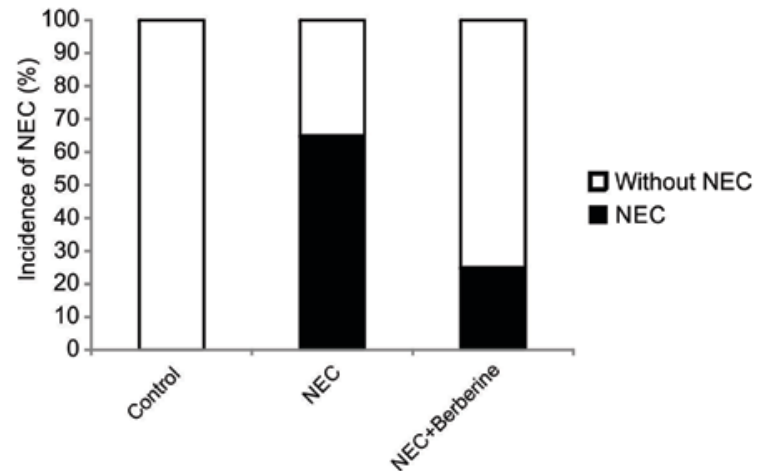

Figure 1. Berberine treatment decreases the incidence of NEC. NEC was confirmed by histological evaluation. Moderate, intermediate and severe tissue damage was considered to be positive indicators of NEC. NEC, necrotizing enterocolitis.

A

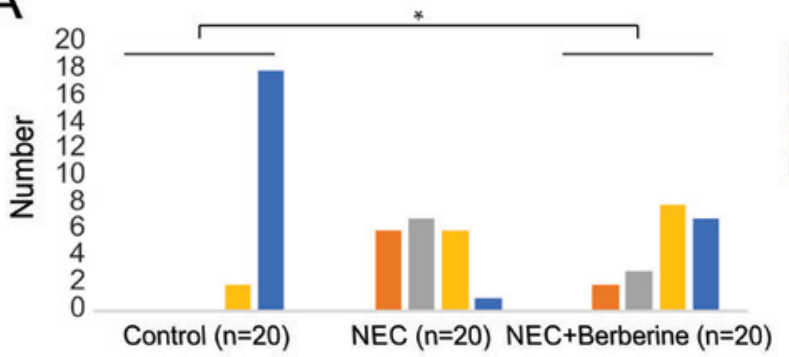

B

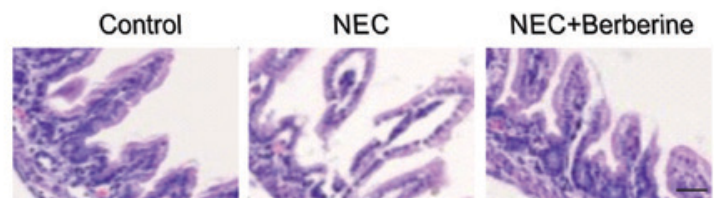

Figure 2. Berberine treatment decreases the severity of NEC. (A) Rats were assessed for tissue damage and NEC grades of M, I or S were recognized as positive indicators of NEC. (B) Hematoxylin and eosin staining of the terminal ilea. Scale bar, $100 \mu \mathrm{m}$. ${ }^{*} \mathrm{P}<0.05$. NEC, necrotizing enterocolitis; $\mathrm{S}$, severe; I, intermediate; M, moderate; L, low; N, normal

the NEC + berberine group, TLR4 mRNA and protein were significantly and markedly suppressed, respectively, compared with the NEC group (Fig. 3).

Berberine treatment decreases cytokine expression in ileal tissues. In the NEC group, NF- $\kappa \mathrm{B}$, iNOS and TNF- $\alpha$ mRNA expression was significantly increased at $48 \mathrm{~h}$ compared with the control group (Fig. 4A). However, in the NEC + berberine group, NF- $\kappa \mathrm{B}$, iNOS and TNF- $\alpha$ mRNA expression was significantly decreased compared with the NEC group. Similarly, NF-кB and iNOS protein levels were increased in the NEC group, while these levels were decreased following berberine treatment (Fig. 4B).

Berberine treatment decreases $I L-6$ and IL-10 expression in the ileum and serum. In the NEC group, the ileal expression of IL- 6 and IL-10 was significantly increased at $48 \mathrm{~h}$ compared with the control group (Fig. 5A). However, in the NEC + berberine group, ileal IL-6 and IL-10 were signifi-
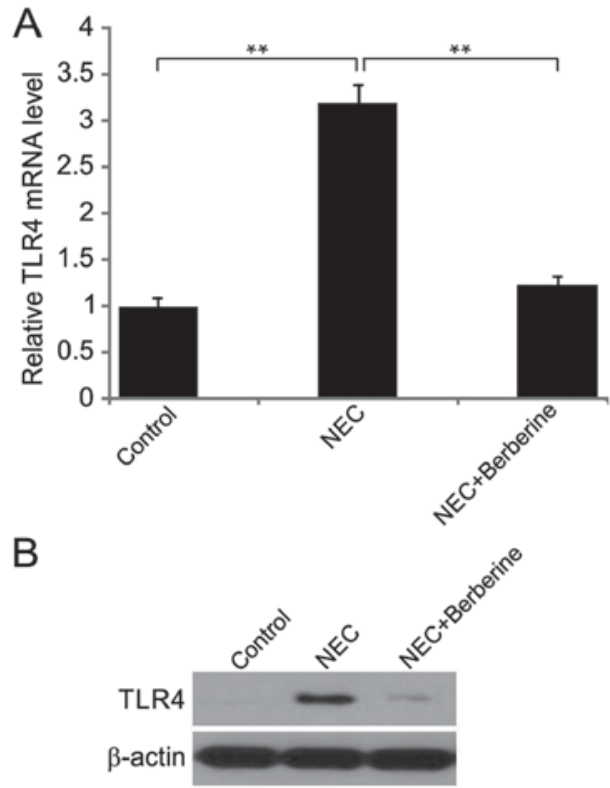

Figure 3. Berberine treatment decreases TLR4 expression in ileum tissues. TLR4 (A) mRNA and (B) protein expression following $48 \mathrm{~h}$ berberine treatment. the results are expressed as mean \pm standard deviation $(n=3)$. ${ }^{* *} \mathrm{P}<0.01$. NEC, necrotizing enterocolitis; TLR, Toll-like receptor.

cantly lower compared with the NEC model group. Similar results were observed in serum samples (Fig. 5B).

Berberine treatment increases the expression of MUC2 and SIgA in ileal tissues. Compared with the control group, the expression level of MUC2 and SIgA protein was significantly lower in the NEC group at $48 \mathrm{~h}$ (Fig. 6). However, in the NEC + berberine group, the expression of MUC2 and SIgA were significantly greater compared with the NEC group.

\section{Discussion}

NEC is a common disorder that affects newborns, primarily occurring in preterm infants of very low birth weight (14). In the present study, NEC symptoms were evident 2 days after the model was established. There are differences in the clinical manifestation of NEC; mild symptoms, including a swollen abdomen and diarrhea, can rapidly develop into necrosis of the intestinal wall, perforation or peritonitis $(2,8)$. Therefore, prevention is particularly important. Studies have suggested several preventive measures, including the use of probiotics to regulate intestinal microecology, intestinal supplementation with arginine and glutamine and treatment with glucocorticoids $(21,22)$. However, the efficacy of these treatments has not yet been proven and their use remains controversial. Therefore, the discovery of safe and effective preventive methods has become a research hotspot.

Symbiotic bacteria often colonize the sterile gut of neonates, which can increase the incidence of NEC (23). TLRs, cell transmembrane receptors of the natural immune system, are critical for pathogen resistance (24). Berberine has been demonstrated to effectively reduce inflammatory cytokine levels (25) and to attenuate NEC by inhibiting inflammation and apoptosis via the phosphatidylinositol 4,5-bisphosphate 3-kinase/RAC- $\alpha$ serine/threonine-protein kinase signaling 

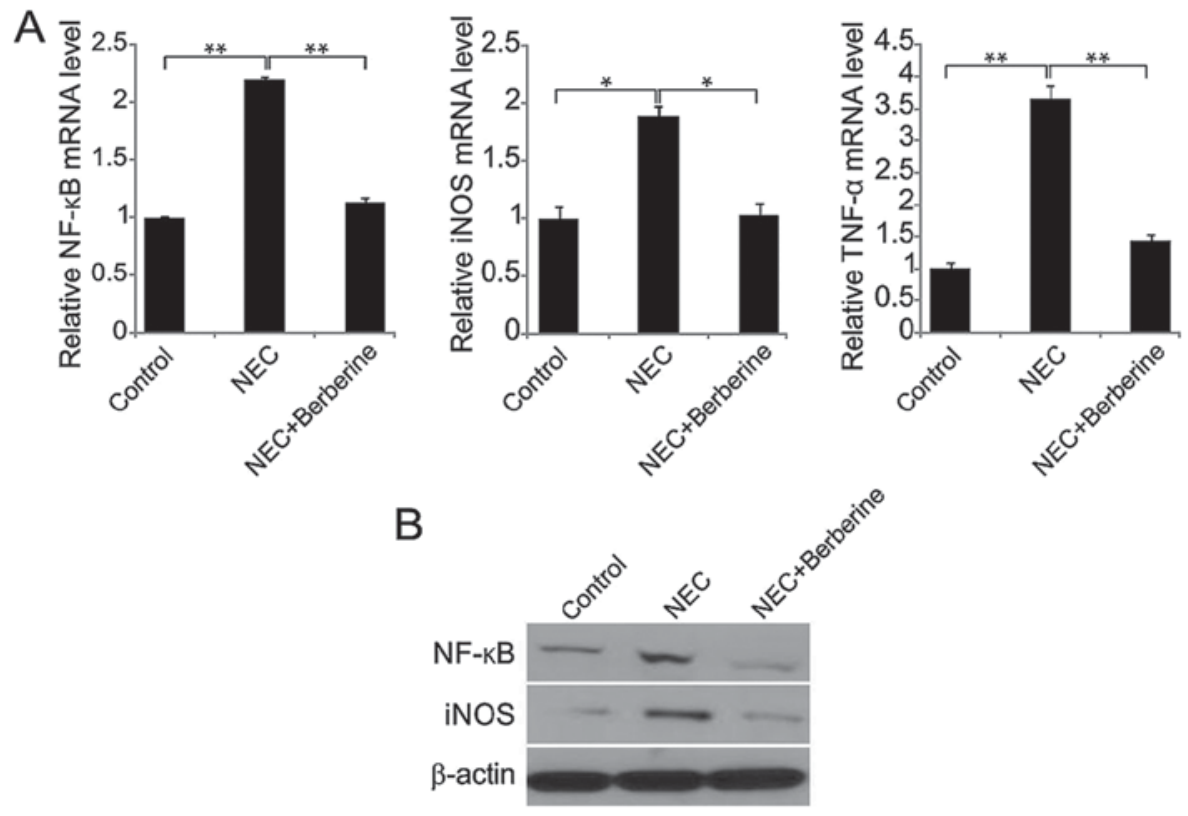

Figure 4. Berberine treatment decreases cytokine expression in the ileum. (A) Relative NF- $\kappa \mathrm{B}$, iNOS and TNF- $\alpha$ mRNA expression following $48 \mathrm{~h}$ berberine treatment. (B) NF- $\mathrm{kB}$ and iNOS protein expression following $48 \mathrm{~h}$ berberine treatment. Results are expressed as the mean \pm standard deviation (n=3). ${ }^{*} \mathrm{P}<0.05$

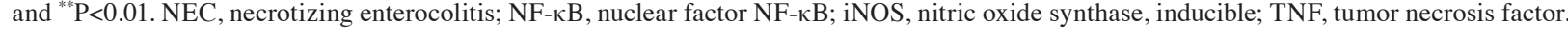
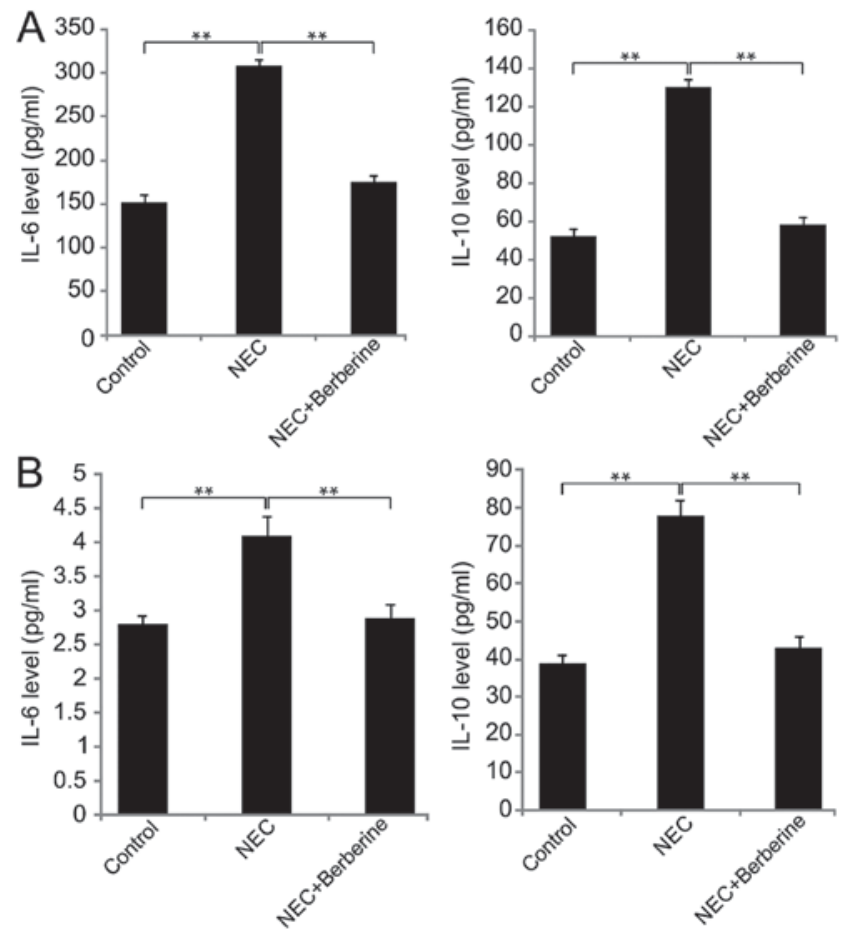

Figure 5. Berberine treatment decreases IL-6 and IL-10 expression in the ileum and serum of rats. (A) Ileal and (B) serum IL-6 and IL-10 expression following $48 \mathrm{~h}$ berberine treatment. (Results are expressed as the mean \pm standard deviation $(n=3) .{ }^{* *} \mathrm{P}<0.01$. NEC, necrotizing enterocolitis; IL, interleukin.

pathway (26). The authors of the present study inferred that berberine may be effective at reducing the incidence and severity of NEC through its anti-inflammatory effects.

NEC is caused by multiple factors that primarily act through the inflammatory cascade (10). A possible target for NEC treatment is the regulation of key components of
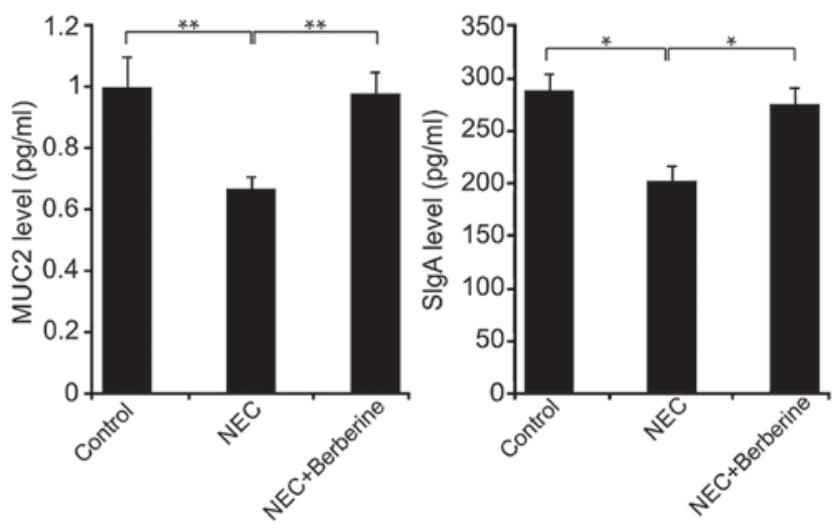

Figure 6. Berberine treatment increases the expression of MUC2 and SIgA in ileal tissues. MUC2 and SIgA protein levels in ileal tissues following $48 \mathrm{~h}$ of treatment. Results are expressed as the mean \pm standard deviation $(n=3)$. ${ }^{*} \mathrm{P}<0.05$ and ${ }^{* *} \mathrm{P}<0.01$. NEC, necrotizing enterocolitis; MUC2, mucin-2; SIgA, RNA polymerase $\sigma$ factor SigA.

the inflammatory response (6). More importance has been attached to the effects of TLR4 in the pathogenesis of neonatal NEC (27). Different TLRs, recognize their respective pathogens, triggering signaling pathways, thereby activating a series of immune responses (28). The p65/p50 dimer of $\mathrm{NF}-\kappa \mathrm{B}$ was identified in nearly all nucleated eukaryotic cells (29). TLR2 and TLR4 are normally lowly expressed in intestinal epithelial cells, and so they have been used to monitor the state of the intestinal flora (24). NF- $\kappa \mathrm{B}$ remains inactive in the cytoplasm until intestinal epithelial cells are stimulated by bacteria acting on the TLRs, triggering the activation of downstream signaling pathways and causing $N F-\kappa B$ to migrate into the nuclei (30). Thereafter, $N F-\kappa B$ has been demonstrated to further promote the overexpression of cytokines associated with immune responses (5). In an LPS-induced animal model of NEC, activated TLR4 triggered an inflammatory cascade, 
thereby regulating intestinal injury repair in neonates and accelerating the development and progression of NEC (4). IL-6 causes neutrophils to recruit and release a large number of active oxygen radicals in lesion areas by virtue of chemotaxis and coordinates with other inflammatory cells to give a cytotoxic effect (31). A positive feedback loop is formed by the stimulation of inflammatory mediators, which can lead to excessive inflammatory responses, injury and necrosis of the intestinal tissues, as well as destruction of the mucosal barrier, thereby developing into NEC (32).

Mucin is a major component of the intestinal mucus layer, covering the surface of the intestinal epithelial cells and acting as the first line of defense (33). Among the 20 known types of mucin, secretory mucin MUC2 was the first to be identified and exists in the highest concentration in the human intestinal cavity (34). MUC2 covers the surface of the intestinal cavity and forms a gelatinous mucus layer to preserve complete barrier function (34). Previous studies have demonstrated that MUC2 is involved in the pathogenesis of NEC (6). SIgA is an immunoglobulin on the surface of intestinal mucosa, which serves an important role in the defense of gastrointestinal mucosa (35). A Previous study has demonstrated that the expression of SIgA was reduced in NEC rats, compared with rats, and that this effect was weakened by Insulin-like growth factor I (7). In the present study, changes were observed in the ileal expression of MUC2. The results indicated that MUC2 and SIgA expression was lower in the NEC group. In the NEC + berberine group, MUC2 and SIgA expression was similar to that observed in the control group.

In summary, the results of the present study demonstrated that enteral administration of berberine ameliorates the clinical symptoms and decreases the incidence of NEC in a neonatal rat model. This may be achieved via berberine-induced TLR4 downregulation, which in turn inhibits the production of inflammatory mediators, and the upregulated expression of MUC2 and SIgA. Together, these expression changes may protect the mechanical and immuno-barrier functions of the intestinal mucosa. Therefore, berberine may be a potential therapeutic agent for the treatment of NEC.

\section{Acknowledgments}

Not applicable.

\section{Funding}

The current study was supported by the Natural Science Foundation of China (grant no. 81460249) and Guizhou Province Joint Fund [qianKehe LH (2015); grant no. 7478].

\section{Availability of data and materials}

The analyzed data sets generated during the present study are available from the corresponding author on reasonable request.

\section{Authors' contributions}

YJ designed and planned the study. YJ and FP collected the data. YJ, FP and YS analyzed the data. JJ interpreted the data. $\mathrm{YJ}$ and $\mathrm{JJ}$ analysed the literature and wrote the manuscript.

\section{Ethics approval and consent to participate}

The present study was approved by the Animal Ethics Committee of The Second People's Hospital of Liaocheng.

\section{Patient consent for publication}

Not applicable.

\section{Competing interests}

The authors declare that they have no competing interests.

\section{References}

1. Hackam DJ and Sodhi CP: Toll-like receptor-mediated intestinal inflammatory imbalance in the pathogenesis of necrotizing enterocolitis. Cell Mol Gastroenterol Hepatol 6: 229-238.el, 2018.

2. Halpern MD and Denning PW: The role of intestinal epithelial barrier function in the development of NEC. Tissue Barriers 3: e1000707, 2015.

3. Dvorak B, Halpern MD, Holubec H, Williams CS, McWilliam DL, Dominguez JA, Stepankova R, Payne CM and McCuskey RS: Epidermal growth factor reduces the development of necrotizing enterocolitis in a neonatal rat model. Am J Physiol Gastrointest Liver Physiol 282: G156-G164, 2002.

4. Livak KJ and Schmittgen TD: Analysis of relative gene expression data using real-time quantitative PCR and the 2(-Delta Delta C(T) ) method. Methods 25: 402-408, 2001.

5. Liu T, Zhang L, Joo D and Sun SC: NF- $\kappa B$ signaling in inflammation. Signal Transduct Target Ther 2, 2017.

6. Martin NA, Mount Patrick SK, Estrada TE, Frisk HA, Rogan DT, Dvorak B and Halpern MD: Active transport of bile acids decreases mucin 2 in neonatal ileum: Implications for development of necrotizing enterocolitis. PLoS One 6: e27191, 2011.

7. Tian F, Liu GR, Li N and Yuan G: YUAN4 insulin-like growth factor I reduces the occurrence of necrotizing enterocolitis by reducing inflammatory response and protecting intestinal mucosal barrier in neonatal rats model. Eur Rev Med Pharmacol Sci 21: 4711-4719, 2017.

8. Garg BD, Sharma D and Bansal A: Biomarkers of necrotizing enterocolitis: A review of literature. J Matern Fetal Neonatal Med 31: 3051-3064, 2017.

9. Rai SE, Sidhu AK and Krishnan RJ: Transfusion-associated necrotizing enterocolitis re-evaluated: A systematic review and meta-analysis. J Perinat Med 46: 665-676, 2018.

10. De Plaen IG: Inflammatory signaling in necrotizing enterocolitis. Clin Perinatol 40: 109-124, 2013.

11. Frost BL, Jilling $\mathrm{T}$ and Caplan MS: The importance of pro-inflammatory signaling in neonatal necrotizing enterocolitis. Semin Perinatol 32: 100-106, 2008.

12. MohanKumar K, Namachivayam K, Chapalamadugu KC, Garzon SA,Premkumar MH, Tipparaju SM and Maheshwari A: Smad7 interrupts TGF- $\beta$ signaling in intestinal macrophages and promotes inflammatory activation of these cells during necrotizing enterocolitis. Pediatr Res 79: 951-961, 2016.

13. Claud EC: Neonatal necrotizing enterocolitis-inflammation and intestinal immaturity. Antiinflamm Antiallergy Agents Med Chem 8: 248-259, 2009.

14. Zani A and Pierro A: Necrotizing enterocolitis: Controversies and challenges. F1000Res 4: F1000 Faculty Rev-1373, 2015.

15. Maheshwari A, Schelonka RL, Dimmitt RA, Carlo WA, Munoz-Hernandez B, Das A, McDonald SA, Thorsen P, Skogstrand K, Hougaard DM, et al: Cytokines associated with necrotizing enterocolitis in extremely-low-birth-weight infants. Pediatr Res 76: 100-108, 2014.

16. Benkoe T, Baumann S, Weninger M, Pones M, Reck C, Rebhandl W and Oehler R: Comprehensive evaluation of 11 cytokines in premature infants with surgical necrotizing enterocolitis. PLoS One 8: e58720, 2013.

17. Habtemariam S: Berberine and inflammatory bowel disease: A concise review. Pharmacol Res 113: 592-599, 2016. 
18. Cicero AF and Baggioni A: Berberine and its role in chronic disease. Adv Exp Med Biol 928: 27-45, 2016.

19. Zhou X, Ren F, Wei H, Liu L, Shen T, Xu S, Wei J, Ren J and Ni H: Combination of berberine and evodiamine inhibits intestinal cholesterol absorption in high fat diet induced hyperlipidemic rats. Lipids Health Dis 16: 239, 2017.

20. Shinyama S, Kaji T, Mukai M, Nakame K, Matsufuji $H$ Takamatsu $\mathrm{H}$ and Ieiri S: The novel preventive effect of Daikenchuto (TJ-100), a Japanese herbal drug, against neonatal necrotizing enterocolitis in rats. Pediatr Surg Int 33: 1109-1114, 2017.

21. Patel RM and Underwood MA: Probiotics and necrotizing enterocolitis. Semin Pediatr Surg 27: 39-46, 2018

22. Harpavat S, Pammi M and Gilger M: Novel treatments for NEC: Keeping IBD in mind. Curr Gastroenterol Rep 14: 373-379, 2012

23. Jiang F, Meng D, Weng M, Zhu W, Wu W, Kasper D and Walker WA: The symbiotic bacterial surface factor polysaccharide A on Bacteroides fragilis inhibits IL-1beta-induced inflammation in human fetal enterocytes via toll receptors 2 and 4. PLoS One 12: e0172738, 2017.

24. Dolasia K, Bisht MK,Pradhan G, Udgata A and Mukhopadhyay S: TLRs/NLRs: Shaping the landscape of host immunity. Int Rev Immunol 37: 3-19, 2018.

25. Mohammadi S, Seyedhoseini FS, Asadi J and Yazdani Y: Effects of berberine on the secretion of cytokines and expression of genes involved in cell cycle regulation in THP-1 monocytic cell line. Iran J Basic Med Sci 20: 530-537, 2017.

26. Fang C, Xie L, Liu C, Fu C, Ye W, Liu H and Zhang B: Berberine ameliorates neonatal necrotizing enterocolitis by activating the phosphoinositide 3-kinase/protein kinase B signaling pathway. Exp Ther Med 15: 3530-3536, 2018.
27. Nanthakumar N, Meng D, Goldstein AM, Zhu W, Lu L, Uauy R, Llanos A, Claud EC and Walker WA: The mechanism of excessive intestinal inflammation in necrotizing enterocolitis: An immature innate immune response. PLoS One 6: e17776, 2011.

28. Dong XS, Xu XY, Sun YQ, Wei-Liu, Jiang ZH and Liu Z: Toll-like receptor 4 is involved in myocardial damage following paraquat poisoning in mice. Toxicology 312: 115-122, 2013.

29. Hong JT: NF-kB as a mediator of brain inflammation in AD. CNS Neurol Disord Drug Targets, 2017.

30. Liu Q, Xu D, Jiang S, Huang J, Zhou F, Yang Q, Jiang S and Yang L: Toll-receptor 9 gene in the black tiger shrimp (Penaeus monodon) induced the activation of the TLR-NF- $\kappa \mathrm{B}$ signaling pathway. Gene 639: 27-33, 2018.

31. Qi W, Shen Q, Zhang L, Han LP and Wang S: Study on the inflammatory intervention of erythropoietin on NEC. Exp Ther Med 11: 2221-2224, 2016.

32. Satoh T, Izumi H, Iwabuchi N, Odamaki T, Namba K, Abe F and Xiao JZ: Bifidobacterium breve prevents necrotising enterocolitis by suppressing inflammatory responses in a preterm rat model. Benef Microbes 7: 75-82, 2016.

33. Sicard JF, Le Bihan G, Vogeleer P, Jacques M and Harel J: Interactions of intestinal bacteria with components of the intestinal mucus. Front Cell Infect Microbiol 7: 387, 2017.

34. Betge J, Schneider NI, Harbaum L, Pollheimer MJ, Lindtner RA, Kornprat P, Ebert MP and Langner C: MUC1, MUC2, MUC5AC and MUC6 in colorectal cancer: Expression profiles and clinical significance. Virchows Arch 469: 255-265, 2016.

35. Chatterton DE, Nguyen DN, Bering SB and Sangild PT: Anti-inflammatory mechanisms of bioactive milk proteins in the intestine of newborns. Int J Biochem Cell Biol 45: 1730-1747, 2013. 